\title{
Uma Metodologia para Teste de Software no Contexto da Melhoria de Processo
}

\author{
Adalberto Nobiato Crespo $^{2,4}$, Odair Jacinto da Silva ${ }^{1}$, Carlos Alberto Borges ${ }^{1}$, Clênio \\ Figueiredo Salviano $^{2}$, Miguel de Teive e Argollo Junior ${ }^{2}$, Mario Jino ${ }^{3}$ \\ ${ }^{1}$ Ampla Consultoria em Informação - Campinas - SP - Brasil \\ ${ }^{2}$ Centro de Pesquisas Renato Archer (CenPRA) - Campinas - SP - Brasil \\ ${ }^{3}$ UNICAMP - Campinas - SP - Brasil \\ ${ }^{4}$ Universidade São Francisco - Itatiba - SP - Brasil \\ \{odair, carlos\} @amplaconsultoria.com.br, \{clenio.salviano, adalberto.crespo, \\ miguel.argollo\} ecenpra.gov.br, jino@dca.fee.unicamp.br
}

\begin{abstract}
Resumo
Uma metodologia para implantação ou melhoria do processo de teste em empresas desenvolvedoras de software foi desenvolvida pelo CenPRA com o objetivo de viabilizar a utilização das práticas de teste pelas empresas. Para experimentação e validação desta metodologia, ela foi aplicada em uma micro empresa de software como parte de um projeto de melhoria de processo. Nesta empresa o processo de teste foi executado nos últimos sete projetos realizados. Este artigo apresenta considerações sobre teste no contexto da qualidade de software, a metodologia desenvolvida, uma aplicação prática e conclusões sobre a metodologia.
\end{abstract}

Palavras chave: Qualidade do Processo, Implantação de Programas de Qualidade, e Verificação, Validação e Testes.

\begin{abstract}
A methodology for the implementation or improvement of the test process in software development organizations was developed by CenPRA in order to make feasible the utilization of test practices by the organizations. As an experimentation and validation of this methodology, it was applied on a small software organization as part of a software process improvement project. In this organization, the test process was performed in the last seven projects. This paper presents considerations on testing in the software quality context, the methodology, a practical application and conclusions about the methodology.

Key words: software quality, test process, methodology and process improvement
\end{abstract}

\section{Introdução}

No mundo atual existe uma demanda não satisfeita por software de qualidade. Organizações estão sofrendo fortes pressões para desenvolver sistemas de informação em curtos espaços de tempo. Tais sistemas precisam ser escalonáveis e integrados com outros sistemas existentes ou em desenvolvimento. Os ambientes tecnológicos nos quais estes sistemas são desenvolvidos estão em constante evolução.

Na medida em que o emprego de sistemas de informação pela sociedade cresce ao ponto em que boa parte dos negócios depende cada vez mais de software e computadores, passa a ser de 
vital importância contar com software de qualidade - software que fornece resultado correto quando alimentado com dados válidos e que identifica corretamente dados de entrada inválidos.

Para o desenvolvimento de software com qualidade, dentro de prazos e custos controlados e compatíveis com o mercado, é fundamental a melhoria dos processos da engenharia de software. Para tanto abordagens e experiências para a melhoria de processo de software baseadas em modelos têm sido utilizadas com sucesso pelas organizações de software. Os modelos mais utilizados têm sido o SW-CMM, ISO/IEC 12207, ISO/IEC 15504 e CMMI. Estes modelos identificam processos fundamentais para a engenharia de software. Todos eles identificam, direta ou indiretamente, teste de software com um destes processos. Teste é fundamental para a avaliação do software desenvolvido. Entretanto, testar software não é uma atividade trivial, e exige conhecimentos, habilidades e infra-estrutura específicos.

A metodologia do teste de software se reflete atualmente no comportamento das empresas na busca em implantar ou mesmo melhorar o processo de teste utilizado. Ainda que as técnicas de teste de software mais utilizadas foram criadas por volta dos anos 70, as empresas têm uma grande dificuldade com a atividade de teste. Isto pode ser um reflexo da falta de profissionais especializados na área de teste de software ou mesmo da dificuldade em implantar um processo de teste utilizando as técnicas existentes na literatura.

Neste contexto, o CenPRA desenvolveu uma metodologia para implantação ou melhoria do processo de teste em empresas desenvolvedoras de software. Este artigo apresenta considerações sobre teste no contexto da qualidade de software, uma visão geral e resumida da metodologia desenvolvida, uma aplicação prática desta metodologia e conclusões.

\section{Teste e Qualidade de Software}

Existem várias tentativas no sentido de definir a atividade de teste, desde a visão intuitiva sobre teste até uma definição formal [1, 2, 3]. Todas as afirmações, sejam intuitivas ou formais, generalizam uma idéia sobre o que é teste de software e essencialmente conduzem ao mesmo conceito: Teste de software é o processo de executar o software de uma maneira controlada com o objetivo de avaliar se o mesmo se comporta conforme o especificado.

Devido a algumas características próprias do software como flexibilidade para mudanças, complexidade e intangibilidade, o teste não é uma tarefa trivial. A dificuldade em testar software é caracterizada por alguns pontos importantes como: o teste de software é um processo caro; existe uma falta de conhecimento sobre a relação custo/benefício do teste; há falta de profissionais especializados na área de teste; existem dificuldades em implantar um processo de teste; há o desconhecimento de um procedimento de teste adequado; há o desconhecimento de técnicas de teste adequadas; há o desconhecimento sobre como planejar a atividade de teste; e finalmente, a preocupação com a atividade de teste somente na fase final do projeto.

Deve ser ressaltado que a atividade de teste exige conhecimento, planejamento, projeto, execução, acompanhamento, recursos e também uma grande interação com as outras equipes.

$\mathrm{Na}$ elaboração do planejamento do teste, uma das etapas é a elaboração da estratégia de teste. A estratégia de teste compreende a definição dos seguintes itens: O nível de teste, isto é, a definição da fase do desenvolvimento do software em que o teste será aplicado; A técnica de teste a ser utilizada; O critério de teste a ser adotado; O tipo de teste a ser aplicado no software.

O nível de teste depende da fase do desenvolvimento do software em que o teste poderá ser aplicado, compreendendo a codificação dos módulos do sistema - Teste de Unidade; a integração 
dos módulos do sistema - Teste de Integração; atendimento aos requisitos funcionais e não funcionais do sistema - Teste de Sistema; aceitação do sistema pelo usuário - Teste de Aceitação; e, finalmente, o Teste de Regressão que é aplicado na fase de manutenção do sistema.

Geralmente a definição da fase do desenvolvimento do sistema em que serão aplicados os testes depende da política de teste da empresa. Por exemplo, uma empresa poderá adotar a política de fazer somente o teste de sistema, ou seja, aplicar o Teste de Sistema depois que os módulos estiverem todos já integrados e não aplicar o Teste de Unidade e Teste de Integração.

A escolha da técnica de teste depende também da fase de desenvolvimento em que o teste será aplicado. Uma técnica de teste direciona a escolha de critérios para geração de casos de teste que, ao serem executados, vão exercitar os elementos requeridos pela abordagem do teste.

Existem, basicamente duas técnicas de teste: Teste Estrutural - técnica de teste que adota critérios para a geração dos casos de teste com a finalidade de identificar defeitos nas estruturas internas do software, através de situações que exercitem adequadamente todas as estruturas utilizadas na codificação; Teste Funcional - técnica de teste que adota critérios para a geração dos casos de teste com a finalidade de garantir que os requisitos do software que foi construído sejam plenamente atendidos.

Ao se adotar uma técnica de teste (Funcional ou Estrutural) é necessário escolher um critério para a elaboração dos casos de teste com a finalidade de testar os elementos do software.

Normalmente os elementos de um software que podem ser testados são: as linhas de comando; as funções implementadas; as variáveis definidas no software; os loops existentes no software; todos os ramos de uma decisão; e os requisitos do software.

O critério de teste serve para orientar o testador na geração dos casos de teste. Os elementos requeridos de um critério de teste são os elementos ou as características do software que deverão ser exercitados quando o software for testado. Os casos de teste gerados devem exercitar os elementos ou as características do software definidos por aquele critério.

Os tipos de teste referem-se às características do software que podem ser testadas, e compreende: Teste de Funcionalidade; Teste de Interface; Teste de Desempenho; Teste de Carga (Stress); Teste de Usabilidade; Teste de Volume; Teste de Segurança.

A Figura 1 ilustra graficamente a ligação existente entre os níveis de teste, as técnicas de teste, os tipos de teste e os critérios de teste que podem ser adotados ao se definir uma estratégia de teste.

\section{Metodologia de Teste}

O Centro de Pesquisas Renato Archer - CenPRA, tem sido procurado por empresas desenvolvedoras de software e mesmo órgãos do governo para realizar projetos dentre os quais a atividade de teste tem seu lugar de destaque.

Visando atender as necessidades dessas empresas o CenPRA, através do grupo de teste da Divisão de Melhoria de Processos de Software - DMPS, desenvolveu um projeto que teve como objetivo criar uma metodologia para a introdução ou melhoria do processo de teste de software em empresas produtoras de software, englobando técnicas, procedimentos e ferramentas, capacitando-as a desenvolver produtos de melhor qualidade[4, 5]. A metodologia está fundamentada na adoção de um processo de teste e nos artefatos sugeridos pela Norma IEEE 829-1998 [6], que descreve os documentos que devem ser gerados na atividade de gerência do teste de software. A metodologia de teste foi projetada e desenvolvida de uma forma que as empresas pudessem instanciar o processo de teste de acordo com as suas necessidades e 
disponibilidade de recursos. Além disso, a metodologia de teste pode ser aplicada a qualquer tipo de software, seja ele sistema de informações ou software científico. Nesta metodologia, a implantação do processo de teste envolve um conjunto de atividades que vai desde o levantamento das necessidades da empresa, passa pela realização de treinamentos da equipe técnica e vai até ao acompanhamento dos trabalhos realizados, constituindo assim, um completo ciclo de implantação da atividade de teste dentro da empresa.

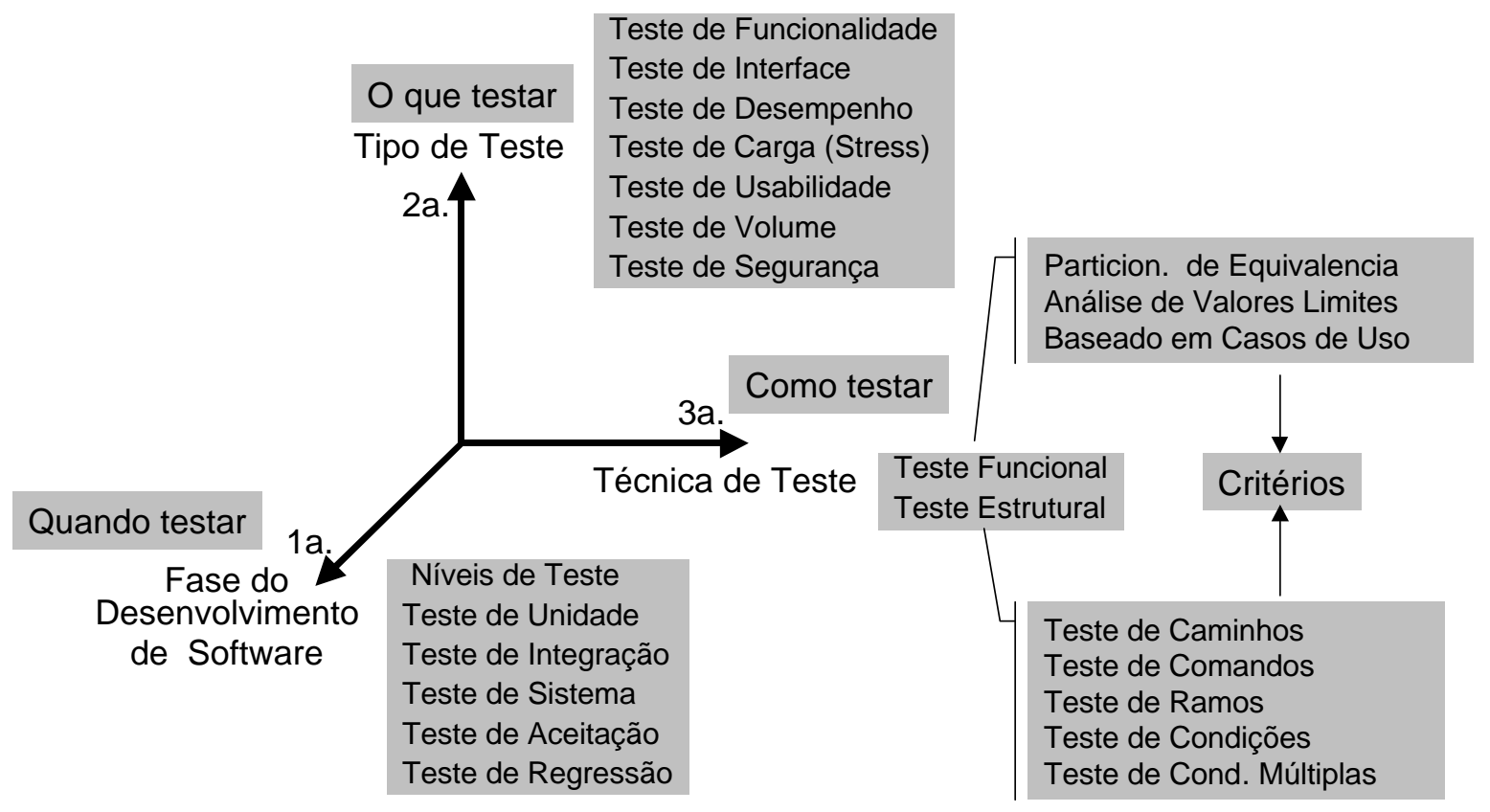

Figura 1 - Relação entre níveis, tipos e técnicas de teste

A metodologia, que preconiza a realização de testes sistemáticos, pode ser empregada tanto por empresas que desenvolvem quanto por empresas que adquirem software, e está dividida em 3 componentes: Treinamento, Processo de Teste e Suporte para Geração de Documentos:

1. Treinamento: Através de cursos, consiste da capacitação em conceitos básicos sobre teste de software, técnicas de teste, documentação de teste e processo de teste. Estes cursos estão divididos em módulos e sua aplicação pode ser adaptada às necessidades específicas de cada empresa.

2. Processo de Teste: A metodologia define um processo genérico de teste que prevê a realização das atividades de planejamento, projeto, execução e acompanhamento dos testes de unidade, integração, sistemas e aceitação. A partir deste processo genérico cada empresa deve instanciar um processo específico que melhor atenda suas necessidades.

3. Suporte para Geração de Documentos: Consiste da aplicação de uma técnica para a criação de documentos que serão utilizados para a gerência do processo de teste, tanto na fase de preparação para a atividade de teste quanto na fase de registro dos resultados do teste. Este componente da metodologia está baseado na Norma IEEE 829-1998, que descreve um conjunto de 8 documentos que cobrem as tarefas de planejamento, especificação e registro 
das atividades de teste de um produto de software. Esta norma pode ser usada para o teste de qualquer tipo de software, seja ele comercial, científico ou militar, sendo definida de forma independente de técnicas, métodos, estratégias, recursos e ferramentas de teste.

O processo de teste instanciado pode ser considerado como o processo de teste empregado de forma global para todos os projetos da organização ou especificamente para cada um de seus projetos. A seção do Plano de Teste que trata a estratégia a ser empregada em cada projeto deve fazer referência ao processo empregado.

O processo de teste proposto na metodologia está baseado em alguns pressupostos básicos:

- Os testes de sistema e aceitação são projetados e executados sob a responsabilidade da equipe de teste.

- Os testes de sistema, e eventualmente também o de aceitação, são realizados de forma iterativa, havendo, antes do início de cada ciclo de teste, uma avaliação rápida do produto.

O processo de teste não contempla automação do teste, medida tomada para manter a descrição inicial do processo simples.

Uma organização pode adotar outros pressupostos, devendo realizar as alterações necessárias no processo de teste instanciado.

Uma premissa básica da metodologia de teste proposta é que o processo de teste, quando adequadamente definido, pode ter um impacto positivo nos resultados de diversas outras atividades de desenvolvimento. Desta forma, o enfoque das atividades de teste não é somente identificar problemas, mas principalmente prevenir problemas. Estas premissas estão presentes em diversas referências sobre teste preventivo $[1,7]$.

$\mathrm{O}$ fato do processo genérico proposto não contemplar a automação de teste reflete a visão de que um processo só deve ser suportado por ferramentas quando estiver convenientemente definido e consistentemente adotado. Zallar afirma que o processo de automação de teste tem maior probabilidade de ser bem sucedido para organizações que possuam uma equipe de teste bem definida e com um processo padrão de documentação seguido [8].

\section{1 - Visão Geral da Norma IEEE 829}

A Norma IEEE 829 descreve um conjunto de documentos para as atividades de teste de um produto de software.

Os 8 documentos definidos pela norma, que cobrem as tarefas de planejamento, especificação e relato de testes, são apresentados a seguir.

Plano de Teste - Apresenta o planejamento para execução do teste, incluindo a abrangência, abordagem, recursos e cronograma das atividades de teste. Identifica os itens e as funcionalidades a serem testados, as tarefas a serem realizadas e os riscos associados com a atividade de teste.

A tarefa de especificação de testes é coberta pelos 3 documentos seguintes.

Especificação de Projeto de Teste - Refina a abordagem apresentada no Plano de Teste e identifica as funcionalidades e características a serem testadas pelo projeto e por seus testes associados. Este documento também identifica os casos e os procedimentos de teste, se existirem, e apresenta os critérios de aprovação. 
Especificação de Caso de Teste - Define os casos de teste, incluindo dados de entrada, resultados esperados, ações e condições gerais para a execução do teste.

Especificação de Procedimento de Teste - Especifica os passos para executar um conjunto de casos de teste.

Os relatórios de teste são cobertos pelos 4 documentos seguintes.

Diário de Teste - Apresenta registros cronológicos dos detalhes relevantes relacionados com a execução dos testes.

Relatório de Incidente de Teste - Documenta qualquer evento que ocorra durante a atividade de teste e que requeira análise posterior.

Relatório-Resumo de Teste - Apresenta de forma resumida os resultados das atividades de teste associadas com uma ou mais especificações de projeto de teste e provê avaliações baseadas nesses resultados

Relatório de Encaminhamento de Item de Teste - Identifica os itens encaminhados para teste no caso de equipes distintas serem responsáveis pelas tarefas de desenvolvimento e de teste.

A norma separa as atividades de teste em três etapas: preparação do teste, execução do teste e registro do teste. A Figura 2 - Relacionamento entre os Documentos de Teste - mostra os documentos que são produtos da execução de cada uma das fases e os relacionamentos entre eles.

Embora a norma possa ser utilizada para o teste de produtos de software de qualquer tamanho ou complexidade, projetos pequenos ou de baixa complexidade podem agrupar alguns documentos propostos, diminuindo o gerenciamento e os custos de produção dos documentos. Além disso, o conteúdo dos documentos também pode ser abreviado.

Adicionalmente, a equipe responsável pelo teste deverá tomar outras decisões em relação à aplicação da norma em projetos específicos, decidindo, por exemplo, se é mais conveniente elaborar um único plano que englobe os testes de unidade, integração e aceitação, ou um plano para cada uma das fases de teste citadas.

Mais do que apresentar um conjunto de documentos, que deve ser utilizado ou adaptado para determinadas empresas ou projetos, a norma apresenta um conjunto de informações necessárias para o teste de produtos de software. Sua correta utilização auxiliará a gerência a se concentrar tanto com as fases de planejamento e projeto quanto com a fase de realização de testes propriamente dita, evitando a perigosa armadilha de só iniciar a pensar no teste de um produto de software após a conclusão da fase de codificação.

\section{2 - Suporte à Implantação do Processo de Teste de Software}

As normas de engenharia de software normalmente são genéricas e demandam um esforço adicional para serem utilizadas com sucesso. Para facilitar a utilização da Norma IEEE-829 a metodologia de teste propõe um método para a implantação do processo de teste de software. Os documentos descritos nesta seção: Guia para Elaboração de Documentos de Teste de Software [9] e Processos para Elaboração de Documentos de Teste de Software [10], formam a base da metodologia proposta. 


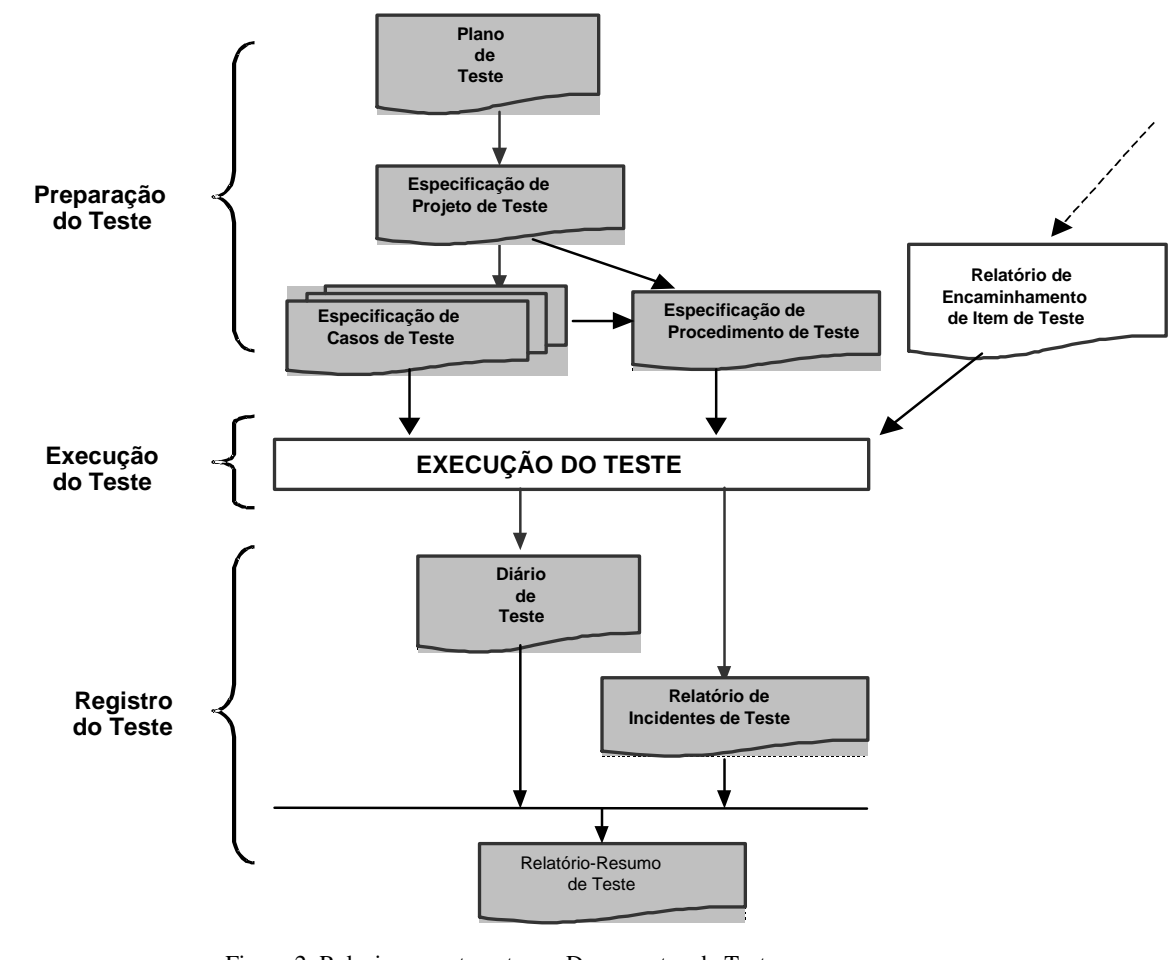

Figura 2: Relacionamento entre os Documentos de Teste

\subsection{1 - Guia para Elaboração de Documentos de Teste de Software}

Esse documento tem o propósito de servir como referência para a criação dos documentos de teste baseados na Norma IEEE 829, tanto na fase de preparação para a atividade de teste quanto na fase de registro dos resultados do teste. O enfoque principal deste guia situa-se na obtenção do conteúdo de cada documento de teste.

\subsection{2 - Processos para a Elaboração de Documentos de Teste de Software}

Este documento apresenta os processos para a elaboração dos documentos de teste de Software baseados na Norma IEEE 829. Os processos abrangem a preparação, a execução e o registro dos resultados do teste e estão descritos segundo o Handbook for Process Management, [11].

Esses processos estabelecem uma orientação geral e, se necessário, podem ser modificados para adequar-se às situações particulares de organizações envolvidas nas atividades de teste.

Um processo é definido para cada documento da norma, segundo a seguinte estrutura:

1. Funções e responsabilidades no processo - participantes na execução das tarefas;

2. Critérios para o início do processo - elementos e/ou condições necessários para iniciar a execução das tarefas;

3. Entradas do processo - dados, recursos ou ferramentas necessários para a execução das tarefas;

4. Tarefas do processo - ações necessárias para produzir as saídas do processo. Para cada tarefa são identificadas suas entradas, com indicação de possíveis fontes, e as saídas produzidas. A 
ordem de apresentação das tarefas não reflete necessariamente a seqüência em que devem ser executadas;

5. Saídas do processo - dados ou produtos gerados pela execução das tarefas;

6. Critérios para término do processo - elementos e/ou condições necessários para encerrar a execução das tarefas; e

7. Medições do processo - medidas a serem coletadas como parte da execução das tarefas.

Dependendo do domínio da aplicação, da estratégia ou da fase de teste, os processos podem ser adaptados de modo a produzir um conjunto maior ou menor de documentos. Contudo, os documentos de preparação para o teste devem incluir: o planejamento do teste, o projeto do teste, os casos de teste e os procedimentos de teste. Além disso, os resultados do teste bem como os incidentes ocorridos durante a execução do teste devem ser adequadamente registrados e condensados num relatório final.

Se necessário, as tarefas ou passos dos processos podem ser estendidos para incluir ações adicionais que podem, eventualmente, resultar em novos documentos e/ou formulários.

Os documentos e os processos neles descritos podem ser aplicados a diversos domínios de aplicação (comercial, científico, etc.), não estando restrita a sua utilização a tamanho, complexidade ou criticalidade do software; podem ser usados para todas as fases de teste, desde o teste de unidade até os testes de aceitação e de regressão.

\section{4 - Aplicação da Metodologia de Teste}

Como parte da experimentação e validação da metodologia de teste, foi realizado um projeto de melhoria de processo em uma micro empresa desenvolvedora de software.

\subsection{Contexto}

A empresa na qual a metodologia foi aplicada é uma pequena empresa de desenvolvimento de projetos de software, fundada em 1995 e instalada em Campinas, SP, nas proximidades da Universidade de Campinas (UNICAMP), no distrito de Barão Geraldo. Esta empresa tem, desde o início de suas atividades, como foco principal o desenvolvimento de projetos de software. Esta área representa cerca de $90 \%$ de seu faturamento mensal. Os seus clientes são, em sua maioria, grandes empresas, multinacionais em muitos casos. Os projetos têm entre 2 e 6 meses de duração e envolvem entre 2 e 4 profissionais.

Neste projeto de melhoria foram tratados vários processos, incluindo o processo de teste. $\mathrm{O}$ projeto de melhoria seguiu a Abordagem de Melhoria de Processo do CenPRA [12]. Esta abordagem suporta a escolha de diferentes modelos de referências e diferentes metodologias para a melhoria de processos específicos. Neste projeto, por exemplo, foi utilizado o modelo de processo da ISO/IEC TR 15504-5 [13] como referência e duas metodologias para dois processos específicos: a metodologia baseada no ProGer para o processo de gerência de projeto e a metodologia de teste do CenPRA para o processo de teste. ProGer é um modelo de processo para gerenciamento de projetos para organizações de desenvolvimento de software de pequeno porte [14].

O projeto foi iniciado em Janeiro de 2002. Seguindo a Abordagem de Melhoria de Processo do CenPRA, o projeto foi executado em seis fases seqüenciais: (1) Início dos Trabalhos e 
Definição de Metas, (2) Avaliação das Práticas Correntes, (3) Planejamento das Ações de Melhoria, (4) Implantação das Ações de Melhoria, (5) Verificação dos Resultados e Aprendizado, e (6) Institucionalização da Melhoria.

A primeira fase foi composta pela elicitação do negócio da empresa, estratégias e objetivos. A escolha da ISO/IEC TR 15504 se deu devido à flexibilidade para adaptações às necessidades da empresa. Decidiu-se utilizar cinco processos da futura norma, para o Nível 2 de capacidade. Os processo escolhidos foram: CUS.2 Supply (Fornecimento), CUS.3 Requirement Elicitation, (Elicitação de Requisitos) MAN.2 Project Management (Gerência de Projeto), ENG.1.6 Software Test (Teste de Software) e ORG.5 Measurement (Medição). Estes processos foram selecionados de forma a cobrir as necessidades de melhoria de processo da empresa.

Uma equipe do CenPRA realizou a avaliação para estes cinco processos, até o Nível 3 de capacidade, para descobrir as práticas utilizadas. A avaliação foi feita de acordo com o método de avaliação criado pelo CenPRA. Os resultados indicaram Nível 2 de capacidade para ORG.5 Measurement e Nível 1 para os outros.

Após a avaliação foram realizadas as outras fazes do projeto. Uma descrição mais detalhada deste projeto de melhoria e de resultados preliminares foram apresentados e discutidos em outras conferências da área $[15,16]$. Este artigo foca apenas a experimentação e validação da metodologia de teste.

\subsection{Situação Atual}

Atualmente existe uma prática institucionalizada na Empresa de planejamento e execução sistemática de teste de sistema, como parte do processo de desenvolvimento de software da empresa.

O fluxograma da Figura 3 descreve uma representação dos elementos principais do processo de teste e seu relacionamento com o processo de desenvolvimento de software, conforme é a sua execução na Empresa.

O processo de teste parte de uma proposta técnica (PPT) bastante detalhada, que associada a um plano de projeto (PP) previamente elaborado, permitem a criação de um plano de teste (PLT).

Dependendo do projeto e nível de exigência do cliente, este deve aprovar o plano de teste. Além de aprovar o plano, o cliente deve se envolver também em sua elaboração, por exemplo através da identificação de critérios de teste.

Após a elaboração do plano de teste (a partir de definição também deste plano), opta-se, em função do tamanho e riscos associados ao projeto, pela elaboração ou não do documento projeto de teste (PRT), que pode iniciar em paralelo ou após a criação do documento de detalhamento de requisitos (DR).

Com o detalhamento de requisitos (DR) finalizado, elabora-se um documento condensando informações sobre casos e procedimentos de teste (CPT), e eventualmente (na ausência de um PRT), questões gerais do projeto de teste, como abordagem do teste por funcionalidade, critérios de aprovação e rejeição, entre outros. Neste ponto o teste está pronto para ser executado. 
Fluxograma das tarefas de implementação e teste, no tempo de vida de um projeto

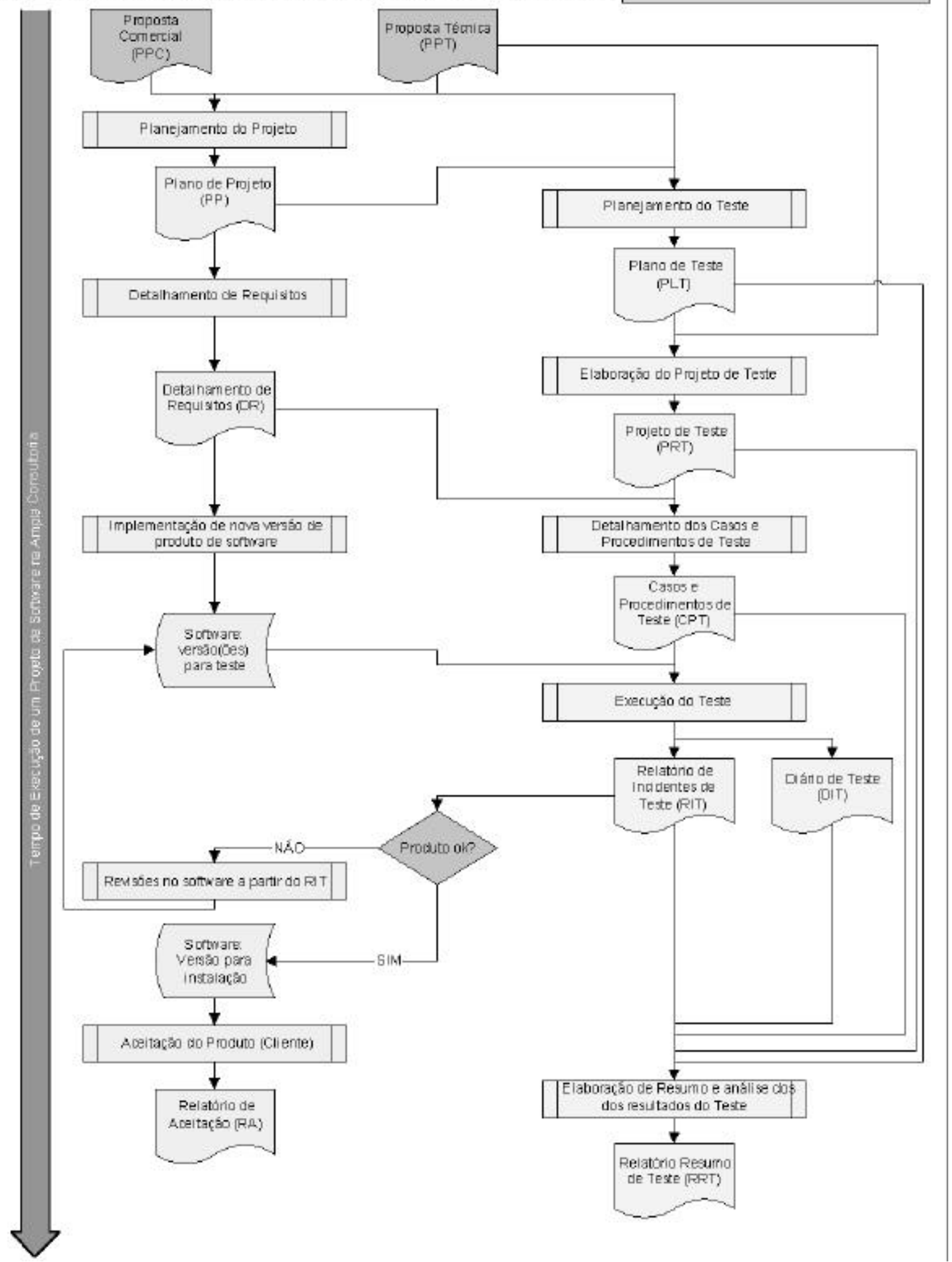

Figura 3: Processo de teste e seu relacionamento com o processo de desenvolvimento 
As tarefas de detalhamento de teste correm simultaneamente com a implementação (codificação) do novo produto de software gerado ou da nova versão contendo melhorias em um software pré-existente.

Assim que uma versão do software é liberada pela equipe de programação, a equipe de teste executa os casos de teste previstos e retorna, via relatório de incidentes de teste (RIT), os problemas detectados à equipe de programação, que deverá corrigi-los.

As novas versões, possivelmente geradas a partir do relatório de incidentes de teste, retornam à etapa anterior, com definição do gerente de teste sobre os casos de teste que deverão ser re-executados até que se tenha um produto considerado adequado para a instalação, conforme decisão do líder do projeto.

Ainda, durante a execução do teste a equipe de teste gera um documento detalhado sobre a execução do teste, denominado diário de teste (DIT). Este documento, permite a geração de análises bem fundamentadas no momento da criação do resumo do teste (RRT), gerado normalmente após a instalação do software aprovado no cliente.

Este documento mostra o esforço necessário para preparação e execução do teste, análises das falhas apontadas, dados gerais do projeto (de teste e de implementação) e as conclusões obtidas.

$\mathrm{Na}$ entrega para o cliente, dispara-se um processo de aceitação do produto gerado, realizado pelos usuários do software após a visita de instalação, com a utilização de um relatório de aceitação (RA), que vai validar não somente o teste do software, mas também a qualidade do produto entregue no sentido da adequação às necessidades específicas de uso.

\subsection{Considerações e dados sobre a utilização do processo}

Este processo já foi utilizado em todos os sete projetos executados após sua implantação. A Tabela 1 mostra um conjunto de dados sobre estes sete projetos, e particularmente, das atividades de teste.

Os dados desta tabela indicam que existe uma prática da execução e gerenciamento de teste e uma base inicial para comparações com projetos futuros buscando identificar problemas e oportunidades de melhoria. Também indicam uma boa execução do processo de medição.

\subsection{Resultados da melhoria}

A melhoria do processo de teste, associada à melhoria do processo de desenvolvimento de software como um todo, permitiu observar os seguintes aspectos, dentre outros.

- Maior controle durante a execução do projeto, devido principalmente ao planejamento prévio e aos pontos de medição, sendo etapas do teste de software alguns dos principais pontos existentes atualmente. Este controle permite ainda que os líderes de projeto nos clientes sejam acionados imediatamente, sempre que houver qualquer desvio em relação ao previsto.

- Estudos e análises, realizadas após o encerramento do projeto e entrega do produto gerado, reforçando os pontos positivos e criando ações corretivas para os pontos negativos detectados no processo como um todo. 
Tabela 1 - Dados sobre os projetos realizados

\begin{tabular}{|c|c|c|c|c|c|c|c|c|c|c|c|c|c|c|c|}
\hline Projet & 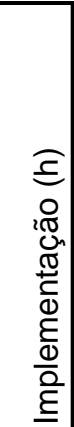 & 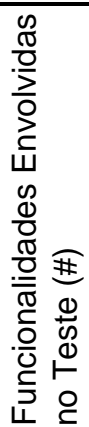 & 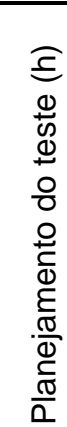 & 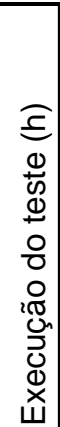 & 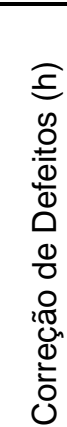 & 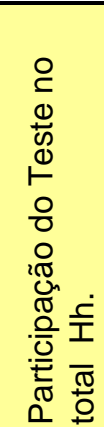 & $\begin{array}{l} \pm \\
\pm \\
0 \\
0 \\
0 \\
0 \\
0 \\
0 \\
0 \\
0 \\
0 \\
0\end{array}$ & 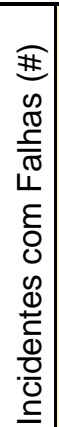 & 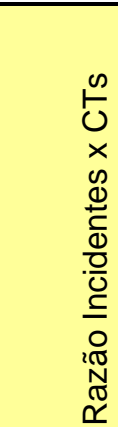 & 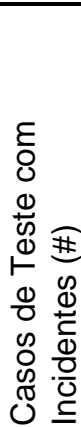 & 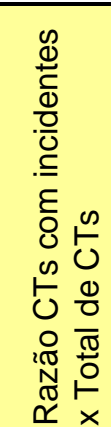 & 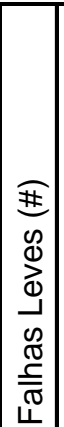 & 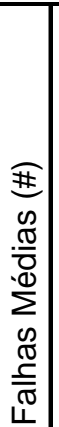 & 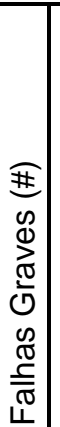 & 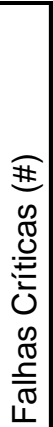 \\
\hline Proj.01 & 176 & 10 & 170 & 90 & 80 & $50,4 \%$ & 220 & 40 & $18,2 \%$ & 75 & $34,1 \%$ & 23 & 5 & 5 & 7 \\
\hline Proj.02 & 7 & 7 & 70 & 61 & 14 & $86,2 \%$ & 113 & 7 & $6,2 \%$ & 14 & $12,4 \%$ & 4 & 2 & 1 & 0 \\
\hline Proj.03 & 85 & 18 & 35 & 25 & 38 & $32,8 \%$ & 42 & 23 & $54,8 \%$ & 20 & $47,6 \%$ & 7 & 11 & 3 & 2 \\
\hline Proj.04 & 4 & 3 & 10 & 6 & 4 & $66,7 \%$ & 19 & 9 & $47,4 \%$ & 9 & $47,4 \%$ & 6 & 2 & 0 & 1 \\
\hline Proj.05 & 17 & 3 & 10 & 9 & 13 & $38,8 \%$ & 26 & 8 & $30,8 \%$ & 17 & $65,4 \%$ & 5 & 1 & 1 & 1 \\
\hline Proj.06 & 10 & 5 & 30 & 17 & 10 & $70,1 \%$ & 25 & 3 & $12,0 \%$ & 10 & $40,0 \%$ & 1 & 0 & 2 & 0 \\
\hline Proj.07 & 90 & 3 & 25 & 32 & 150 & $19,2 \%$ & 31 & 75 & $241,9 \%$ & 25 & $80,6 \%$ & 29 & 9 & 10 & 27 \\
\hline
\end{tabular}

- Qualidade do produto final gerado, no sentido da verificação (requisitos definidos implementados corretamente). No sentido da aceitação, a documentação de projeto completa ajuda a fornecer subsídios para que o usuário possa verificar o aspecto da adequação às necessidades.

- Outro ponto quanto à qualidade é que a documentação gerada permite perceber mais claramente as falhas relacionadas a detalhamentos incorretos ou incompletos de requisitos.

- Os clientes parecem compreender melhor as dificuldades inerentes à produção de software, aumentando o respeito pelas informações transmitidas, documentação apresentada, necessidades explicitadas, aceitando até maiores prazos para entrega de produtos com maior qualidade. A questão de maiores custos associados ainda é uma variável a ser medida.

- Há um aumento nos custos gerais de produção de software, esperados certamente no início da implementação dos novos processos, mas que poderão ser diluídos apenas no longo prazo.

O objetivo da empresa é, a partir dos dados gerados pelo processo de teste, medir sistematicamente cada projeto de software corrigindo desvios e modificando conseqüentemente as prioridades e ações corretivas a serem tomadas; em outras palavras, aprimorando os processos com o aprendizado.

Após a implantação do processo de teste na empresa, as seguintes melhorias foram observadas.

- Existe um menor número de defeitos descobertos após instalação do software, diminuindo de forma significativa os custos e problemas associados a correções urgentes de um sistema em produção.

- A equipe de programação está mais atenta: às tarefas de verificação, antes da liberação de uma versão para teste, causando inclusive uma melhoria no produto, trazendo além de menor desgaste da equipe, menores custos para a empresa. 
- Os clientes, verificando os resultados iniciais obtidos, têm dado mostras significativas que estão dispostos a esperar mais pelo produto final. Mesmo assim, nem sempre aceitam aumento nos custos. Em seguida, eles passam a exigir tal comportamento, em projetos posteriores ao primeiro onde tiveram contato com o processo interno em implantação, ou seja, passam a não querer mais versões do software não devidamente testadas.

- Os testes sistemáticos têm fornecido dados e informações relevantes à melhoria tanto do próprio processo de teste quanto do processo de desenvolvimento como um todo, aumentando sua eficiência e/ou abrangência, e permitindo a criação de pessoal capacitado para as práticas necessárias.

- Os testes sistemáticos têm se mostrado, ainda, um bom ponto de partida para medições, relacionadas ao processo geral de desenvolvimento, ao processo de teste, e ainda a outros processos internos da empresa.

- Pelo lado do investimento, os testes sistemáticos tendem a "se pagar" somente num horizonte de médio e longo prazo. Espera-se que o processo de teste continue em evolução, tanto para seu contínuo aperfeiçoamento quanto, em um prazo mais longo, para a incorporação de ferramentas.

\subsection{Avaliação do processo de teste}

Após a implantação do processo, foi realizada uma avaliação superficial das atividades de teste segundo o processo de teste do modelo exemplo de avaliação de processo da ISO/IEC 15504, chamado de ISO/IEC 15504-5. O resultado desta avaliação foi comparado com uma avaliação realizada em 2001, antes da realização deste ciclo de melhoria. Também foi realizada uma avaliação em relação à área de processo de Verificação do CMMI, que é a área de processo mais próxima do escopo do trabalho em teste.

O resultado da avaliação realizada em 2001 mostrou que a empresa executa o processo de teste no Nível 1 de capacidade. Existiam algumas atividades de teste, sem nenhuma sistemática, nem planejamento. Na avaliação de 2004, existe uma indicação do atendimento do Nível 2 de capacidade, com alguns elementos do Nível 3. Como a avaliação foi superficial, o máximo que se pode dizer é que existem indicadores.

O processo de teste de software da ISO/IEC TR 15504 tem como objetivo "garantir que a implementação de cada requisito do sistema seja testada em conformidade com o requisito e que o sistema esteja pronto para ser liberado". Como resultado de uma implementação com sucesso do processo de teste de sistema em uma organização, a 15504-5 requer que evidências dos seguintes quatro elementos sejam identificados durante uma avaliação:

- um critério para o sistema é desenvolvido que demonstre a conformidade com os requisitos;

- sistema é verificado utilizando o critério definido;

- os resultados do teste são registrados; e

- uma estratégia de regressão é desenvolvida e aplicada para retestar o sistema quando mudanças forem feitas em elementos do sistema.

Para que o processo de teste da organização esteja no Nível 1 de capacidade, o objetivo do processo e os quatro resultados estejam sendo atingidos. Para que este processo também esteja no 
Nível 2, a execução do teste tem que ser planejada e acompanhada, exigindo uma boa gerência do teste, e os principais produtos de trabalho do teste têm que ser identificados, definidos, produzidos e verificados. Para um processo estar no Nível 3 ele deve estar bem definido e ser seguido em cada uma de suas execuções.

$\mathrm{Na}$ avaliação superficial foi verificado que:

- os objetivos do processo de teste estavam sendo atingidos (portanto satisfaz o Nível 1 de capacidade);

- a execução é planejada e acompanhada, e os principais produtos de trabalho (no caso: Plano de Teste PLP, Projeto de Teste PRT, Casos e Procedimentos de Teste CPT, Relatório de Incidentes de Teste RIT, Diário de Teste DIT, e Relatório Resumo de Teste RRT) são identificados, definidos, produzidos e verificados (a verificação não é muito sistematizada, porém sendo uma micro empresa, foi entendido que isto não compromete, neste caso, o Nível 2 de capacidade) (portanto satisfaz o Nível 2 de capacidade); e

- existe alguns aspectos do processo já bem definidos (como por exemplo, fluxo de execução), mas ele ainda não é completo (portanto ainda não satisfaz o Nível 3 de capacidade).

Com isto, o processo de teste de software foi considerado com o Nível 2 de capacidade, em relação à ISO/IEC 15504-5. Para a área de processo de verificação da representação contínua CMMI-SE/SW as indicações também sinalizam o Nível 2 de capacidade.

Está previsto no programa de melhoria da empresa, para 2004, uma avaliação detalhada, seguindo todos os requisitos da ISO/IEC 15504, de um conjunto de processos relevantes da empresa, incluindo o processo de teste.

\section{Conclusão}

Com a aplicação da metodologia em uma micro empresa de desenvolvimento de software, resultando em um processo de teste que foi utilizado com bons resultados em sete projetos e atingiu o Nível 2 de capacidade de processo segundo a Norma ISO/IEC 15504, pode ser concluído que:

- a metodologia é viável de ser aplicada em uma micro empresa, como parte de um programa de melhoria de processo; e

- o processo de teste implantado pela metodologia gera melhorias visíveis aos clientes e aos desenvolvedores, melhorando a qualidade do software e o relacionamento entre a empresa e os clientes.

A metodologia do CenPRA ajuda a estruturar as atividades de teste desde o início de um projeto de desenvolvimento de software, tornando claro para todas as partes envolvidas o relacionamento entre o processo de desenvolvimento de software e as atividades de teste. A definição pela metodologia dos principais artefatos do processo de teste, baseado na Norma IEEE STD 829, é o principal orientador das atividades de teste.

\section{Agradecimentos}

O desenvolvimento e experimentação da metodologia de teste é parte do programa de pesquisa tecnológica do CenPRA em melhoria e avaliação de processo de software. A aplicação da melhoria de processo e da metodologia de 
teste na empresa foi parcialmente financiado pelo programa SEBRAE/FINEP PATME e teve apoio do Núcleo SOFTEX - Campinas.

\section{Referências}

[1] Beizer, B.; Black-Box Testing: techniques for funcional testing of software and system; Wiley, New York, 1995.

[2] Hetzel, B.; The Complete Guide to Software Testing; $2^{\text {nd }}$ Edition, John Wiley \& Sons, 1988.

[3] Myers, G. J.; The Art of Software Testing,; Wiley, New York, 1979.

[4] Crespo, A. N., Martinez, M. R., Jino, M., Argolo, M. T.; Application of the IEEE 829 Standard as a Basis for Structuring the Testing Process; The Journal of Software Testing Professionals, Vol. 3, N. 3, December 2002.

[5] ___ Acceptance Testing of an Outsourced Application: Approach and Documentation Issues; The Journal of Software Testing Professionals, Vol. 4, N . 1, March 2003.

[6] IEEE Computer Society; IEEE Std 829: Standard for Software Test Documentation; September, 1998.

[7] Craig, R. D., Jaskiel, S. P.; Systematic Software Testing; Artech House Publishers, 2002.

[8] Zallar, K.; Are You Ready for Test Automation Game?; STQE - Software Testing and Quality Engineering Magazine, Vol.3, N. .6, Nov/Dec 2001.

[9] Centro de Pesquisas Renato Archer - CenPRA, Divisão de Melhoria de Processos de Software - DMPS; RT - Guia para Elaboração de Documentos de Teste de Software; Relatório Técnico, 2001.

[10] ___ _ RT - Processos para Elaboração de Documentos de Teste de Software; Relatório Técnico, 2001.

[11] Space and Naval Warfare Systems Center San Diego, Software Engineering Process Office, D12; Handbook for Process Management; Version 1.0, San Diego. September, 1998.

[12] Clênio F. S.; Melhoria e Avaliação de Processo de Software com a ISO/IEC 15504 e CMMI; Notas de Aula, Pós-Graduação "Lato Sensu", UFLA/FAEPE, 2003.

[13] ISO/IEC; ISO/IEC TR 15504 Software Process Assessment.1998.

[14] Roullier, A. C.; Gerenciamento de Projetos de Software para Empresas de Pequeno Porte; Tese de Doutorado, UFPE, 2001.

[15] Silva, O. J., Borges, C. A., Salviano, C. F., Sampaio, A. L., Crespo, A. N., Roullier, A. C.; An ISO/IEC 15504-Based Software Process Improvement Project in a Small Brazilian Software Organization;, in Proceedings of SPICE-2003: The Joint ESA - Third International SPICE Conference on Process Assessment and Improvement, Noordwijk, The Netherlands, March 2003.

[16] Silva, O. J., Borges, C. A. Salviano, C. F., Crespo, A. N., Roullier, A. C.; Aplicação da ISO/IEC TR 15504 na Melhoria do Processo de Desenvolvimento de Software de uma Pequena Empresa; Anais do Simpros 2003: Simpósio Internacional de Melhoria de Processo de Software, Recife, Brasil, Novembro 2003. 